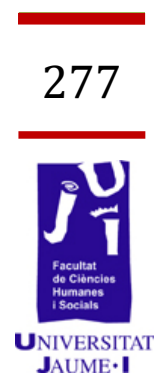

\title{
Relación entre Teoría de la Mente y relaciones entre iguales en alumnado de Primaria
}

\author{
Sandra Carricondo Chavarría \\ al191769@uji.es
}

Clara Andrés Roqueta candres@psi.uji.es 
La investigación acerca de la Teoría de la mente (TM) relacionada con los problemas sociales de los niños/as es escasa (a excepción de aquellos con autismo). Sin embargo, hay niños/as en riesgo de padecer dificultades en esta área cuando manifiestan dificultades de socialización durante el período escolar. Concretamente, distintos autores defienden que los niños/as populares en sus aulas están por encima de la media en las diferentes tareas de la TM y, por el contrario, aquellos/as rechazados/as que están por debajo. Por tanto, como novedad, en el presente estudio se propuso como ampliar los estudios existentes en tres aspectos: establecer comparaciones entre edades y sexo para cada tipo sociométrico (incluyendo niños/as olvidados/as y medios/as), incluir la apreciación de las percepciones (ajuste a la realidad) de cada alumno/a sobre su socialización, y ampliar las tareas mentalistas para caracterizar a cada tipo sociométrico. Por todo ello, el principal objetivo de este estudio comparar el nivel de TM, y los diferentes tipos sociométricos, en una misma muestra de niños/as pertenecientes a aulas ordinarias de primaria (47 alumnos de 20 y 40 de primaria). Para la obtención de los datos se empleó un test sociométrico (para conocer los diferentes tipos sociométricos y variables relativas a las nominaciones y percepciones) y seis clases tareas para la evaluación de la TM. Después de analizar todos los datos obtenidos, se llegó a la conclusión de que los alumnos varones son ligeramente superiores en todas las tareas de la TM; que a mayor edad, mayor TM presenta cada alumno/a; y que los niveles de TM y el ajuste a la realidad de cada tipo sociométrico atienden al patrón de Ignorado<Rechazado<medio<popular. Finalmente, se establecen directrices para la intervención.

Palabras clave: Teoría de la Mente, Tipos sociométricos, relaciones entre iguales, Aulas de primaria

\section{Introducción}

Excepto para aquellos que padecen un Trastorno del Espectro Autista, la investigación acerca de la Teoría de la Mente (TM, a partir de ahora) relacionada con los problemas sociales de los niños/as es escasa. Este es el caso del fenómeno de rechazo entre iguales que existe en las aulas de los centros escolares de Primaria (Villanueva, Clemente y García-Bacete, 2000). Según Cillessen, IJzendoorn, Lieshout y Hartup (1992) se entiende como niños/as rechazados/as en el ámbito escolar a aquellos/as que quedan excluidos de su entorno entre iguales. Tienden a comportarse de forma diferente a sus compañeros/a y, consecuentemente, no se benefician del proceso de sociabilización entre iguales de la misma manera que el resto. Este rechazo comienza a hacerse visible a finales de la etapa de educación infantil (entre los 4 y los 6 años), y se va acentuando a medida que avanzan en la escolaridad obligatoria (Hay, Payne y Chadwick, 2004). Además, se ha demostrado que el rechazo recibido en la escuela puede 
conllevar efectos negativos a lo largo del desarrollo del niño, tales como el abandono de los estudios, ansiedad o fracaso escolar, además de ser más propensos a la soledad (Asher y Wheeler, 1985).

Actualmente, se reconoce que el rechazo entre iguales en la escuela es un problema que afecta entorno al $15-20 \%$ del alumnado de un aula, y suele ser una característica fundamentalmente asociada a los varones (García-Bacete, Sureda y Monjas, 2010). Además, investigaciones recientes han demostrado que la comprensión social (ligada a la TM) va a estar limitada en cierta manera, dado que esta sociabilización positiva no se desarrolla del mismo modo que en el resto del aula.

Una buena TM es muy útil a la hora de desenvolverse en la sociedad, y sobre todo, para ser aceptados por las personas que nos rodean. Evolutivamente, es aceptado que los/las niños/as de entre 3 y 5 años van adquiriendo esta capacidad para predecir y explicar el comportamiento y los sentimientos de los demás en función de la comprensión de sus estados mentales tales como creencias, deseos y/o percepciones sobre la realidad (Astington, 1993; Wellman, 1990, leído en Slaughter, Dennis y Pritchard, 2002). Los niños no adquieren la TM de forma innata, sino la incorporan y ejercitan mediante la interacción con otras personas. Para que este aprendizaje se produzca correctamente, es necesaria la colaboración de un entorno social activo y participativo en actividades socioculturales, así como la orientación de adultos más expertos o de sus iguales (Hay y otros, 2004). Si las personas que orientan este aprendizaje tienen una TM desarrollada, el aprendizaje se producirá más rápido, mientras que si poseen una TM menos competente, el aprendizaje será menos efectivo. Por tanto, un ambiente rico que proporcione las interacciones sociales positivas va a permitir que el niño intente entender la mente de otras personas y adquiera la capacidad de empatizar con ellas y ponerse en su lugar, hecho fundamental para poder sociabilizarte correctamente (Hughes y Leekam, 2004). Por el contrario, no tener en consideración los pensamientos y sentimientos de los demás (falta de TM) perjudicaría una correcta sociabilización, y un niño/a podría pasar a ser socialmente rechazado por sus iguales.

En este sentido, se puede producir un bucle en el que los/las niños/as rechazados se ven atrapados: normalmente estos/as niños/as están solos/las en el contexto escolar, juegan solos/las y no interactúan con nadie en los patios, ya que sus iguales les privan de poder experimentar un trato social. Así, pierden oportunidades para ejercitar y entrenar su TM, es decir, aprender cómo se debe interactuar, qué es lo que al resto de compañeros les molesta, cómo se sienten, etc.

\section{Niveles de TM en distintos tipos sociométricos}

Autores como Watson, Nixon y de Capage (1999) apoyan la teoría de que la comprensión de la TM viene muy ligada a los déficits sociales sufridos por los/las niños/as rechazados/as. Concretamente, aunque pocos, algunos investigadores han estudiado el nivel de TM en los/las 
niños/as rechazados y otros tipos sociométricos con problemas de socialización en el aula, así como aquellos a los que se les pueda atribuir un mejor manejo de las habilidades socio-emocionales con sus iguales (ej. Líderes o Populares). En la Tabla 1 se ofrece un resumen de los principales estudios sobre los tipos sociométricos "Rechazado" y "Popular", haciendo especial énfasis en la edad de los participantes y las tareas de TM empleadas. Por un lado, respecto a las tareas de TM, se puede observar que los distintos estudios emplean diversas tareas clásicas para evaluar esta habilidad mentalista tan compleja: tareas de Creencias Falsas de 1er orden y 2o orden, tarea del Engaño, tareas de Emociones, Tarea de Historias Extrañas, o Meteduras de Pata e Historias Morales.

Tabla 1. Estudios que versan sobre Teoría de la Mente (TM) en distintos tipos sociométricos.

\begin{tabular}{|c|c|c|c|c|c|c|}
\hline \multirow[b]{2}{*}{$\begin{array}{l}\text { Estudio (edad } \\
\text { muestra) }\end{array}$} & \multirow[b]{2}{*}{ Tipo } & \multicolumn{5}{|c|}{ TAREAS DE TM } \\
\hline & & Engaño & CF & Emociones & $\begin{array}{l}\text { Has } \\
\text { Extrañas }\end{array}$ & $\begin{array}{c}\text { M. pata / } \\
\text { Has } \\
\text { Morales }\end{array}$ \\
\hline $\begin{array}{l}\text { Peterson y Siegal, } \\
2002 \text { (4-5 años) }\end{array}$ & Popular & - & $>$ & - & - & $>$ \\
\hline $\begin{array}{l}\text { Slaughter y otros, } \\
2002 \text { (4-6 años) }\end{array}$ & Popular & - & $>$ & - & - & - \\
\hline $\begin{array}{l}\text { Villanueva y otros, } \\
2000 \text { (4-7 años) }\end{array}$ & Popular & $\begin{array}{c}> \\
\text { (chicas) }\end{array}$ & $=$ & $=$ & $=$ & - \\
\hline $\begin{array}{l}\text { Sutton y otros, } \\
1999 \text { (7-10 años) }\end{array}$ & $\begin{array}{c}\text { Popular } \\
\text { "manipulador" } \\
\text { (bully) }\end{array}$ & & $>$ & $>$ & $>$ & $>$ \\
\hline $\begin{array}{c}\text { Sutton y otros, } \\
1999 \text { (7-10 años) }\end{array}$ & $\begin{array}{c}\text { Rechazado } \\
\text { (víctima) }\end{array}$ & & $<$ & $=$ & $<$ & $<$ \\
\hline $\begin{array}{l}\text { Happé y Frith, } \\
1996 \text { (6-12 años) }\end{array}$ & $\begin{array}{c}\text { Rechazado } \\
\text { agresivo / } \\
\text { inadaptado }\end{array}$ & & $=$ & - & - & - \\
\hline $\begin{array}{l}\text { Villanueva y otros, } \\
2000 \text { (4-7 años) }\end{array}$ & Rechazado & $=$ & $=$ & $=$ & $\begin{array}{c}< \\
\text { (mentira } \\
\text { piadosa; } \\
\text { chicos) }\end{array}$ & - \\
\hline $\begin{array}{l}\text { Slaughter y otros, } \\
2002 \text { (4-6 años) }\end{array}$ & Rechazado & - & $\begin{array}{c}< \\
\begin{array}{c}\text { (lenguaje } \\
\text { bajo) }\end{array} \\
\end{array}$ & - & - & - \\
\hline $\begin{array}{l}\text { Peterson y Siegal, } \\
2002 \text { (4-5 años) }\end{array}$ & Rechazado & - & $\begin{array}{c}< \\
(\sin \\
\text { amistades })\end{array}$ & - & - & $\begin{array}{c}< \\
(\sin \\
\text { amistades })\end{array}$ \\
\hline $\begin{array}{c}\text { Banerjee y } \\
\text { Watling, } 2005 \text { (5-6 } \\
\text { años) } \\
\text { Banerjee y otros, } \\
2011 \text { (8-9 años) }\end{array}$ & Rechazados & & & - & - & $<$ \\
\hline
\end{tabular}


En relación a cuál es la competencia en dichas tareas de los niños con los tipos sociométricos, la Tabla 1 ofrece información sobre aquellos ubicados en las categorías "rechazado" y "popular": en primer lugar, por lo que respecta al tipo sociométrico "Rechazado", en general, la mayoría de estudios coinciden en que presentan una TM inferior a la media. Concretamente, se detectan problemas en tareas de CF, historias extrañas, historias morales y meteduras de pata. Sin embargo, presentan niveles similares a sus iguales no-rechazados en tareas sobre reconocimiento de emociones y de engaño. En segundo lugar, en relación al tipo sociométrico "Popular", ocurre al contrario que en los/las niños/as rechazados/as, puesto que se observa que tienen una TM superior a la media en la mayoría de las tareas explicadas en párrafos anteriores. Además, un dato muy interesante a destacar del estudio de Villanueva y otros (2000), es cómo el sexo de los alumnos rechazados y populares influye en el desarrollo de algunas habilidades mentalistas. Específicamente, se demuestra que las niñas líderes desempeñaron mejor la tarea de engaño (pero no los niños líderes, que se encontraron en la media), y que los niños rechazados tuvieron especial dificultad con la historia de "mentira piadosa" de las Historias Extrañas (pero no las niñas rechazadas, que se encontraron en la media). La Tabla 1 también incluye un estudio semejante sobre un fenómeno relacionado (aunque distinto) al que aborda el presente trabajo, como es el caso del bullying. Este estudio realizado por Sutton, Smith y Swettenham (1999), proponen que el "acosador" (bully) posee una TM superior que le permite manipular fácilmente al resto y, por el contrario, los/las niños/as "víctimas" de los compañeros/as tenían un nivel más bajo. En este caso, el "acosador" podría considerarse un líder, y la persona "víctima" rechazada.

Por otro lado, trabajos posteriores comienzan a emplear otras tareas novedosas, como aquellas que valoran la capacidad para detectar el humor gráfico. La evaluación de dicha habilidad es considerada como el camino "no verbal" para conocer las habilidades de TM en niños y niñas (PucheNavarro y Lozano, 2002), ya que TM y humor poseen en común la comprensión de la relación inferencial para hallar una incongruencia. Concretamente, para detectar humor representado gráficamente, PucheNavarro (2004) define la necesidad de poseer otras habilidades, a parte de la TM, como por ejemplo: ser capaz de detectar la información no explícita ni presente en la viñeta (habilidades de tipo analógico y representacional), o saber elegir la alternativa incongruente frente al resto de alternativas sin atractivo lúdico (donde entrarían en juego habilidades de función ejecutiva y memoria de trabajo para establecer comparaciones, retener información, enfatizar y omitir detalles, etc.). Sin embargo, sumado al hecho de que los trabajos sobre humor gráfico son novedosos, no se conocen hasta el momento estudios que hayan empleado relacionándolas con el estatus social de los niños/as.

Para un docente es de gran utilidad saber la estructura social de los grupos que tiene a su cargo. Sabiendo los datos sociométricos de su aula, puede guiar a los/las niños/as y, por ejemplo, intentar relacionar más a los 
sujetos rechazados con líderes para intentar paliar el desnivel jerárquico social. A parte, si se demuestra que el/la rechazado/a tiene de una TM por debajo del nivel que sus compañeros/as, se podría intervenir de manera que se mejore el conocimiento de estos estados mentales donde "flojean" más, para prevenir un posible rechazo escolar. Por todo ello, en el presente estudio se pretende continuar y ampliar la información hallada en los trabajos anteriores, intentando mejorar los siguientes aspectos: a) Edades distintas y control del sexo; b) Ampliación de la cantidad de tareas de TM en la misma muestra y en distintos tipos sociométricos; y c) Estudio de la relación de la TM con las percepciones positivas/negativas acertadas, y las falsas percepciones.

\section{Objetivos}

En base a las necesidades expuestas, los objetivos del presente estudio se dividen en:

1. Estudiar la TM de manera global en distintos rangos de edad, establecer diferencias entre sexos, y entre tipos sociométricos. En este sentido, se pretende explorar el perfil de TM (replicando algunos de los aspectos ya explorados, y de los tipos "rechazado" y "popular") con tareas de CF de segundo orden, emociones secundarias, sentido del humor y meteduras de pata.

2. Estudiar el ajuste a la realidad de su socialización de los distintos tipos sociométricos: percepciones acertadas/falsas y nominaciones inesperadas positivas y negativas.

A partir de los objetivos propuestos, y en base a los estudios previos expuestos en la introducción, las hipótesis del presente trabajo experimental son:

-Hipótesis 1. Según el estudio de Villanueva y otros (2000) el sexo está relacionado con la adquisición de la TM, puesto que las niñas líderes desempeñaron mejor algunas tareas mentalistas que los niños líderes, y los niños rechazados obtuvieron menor competencia que las niñas rechazadas en otras. Como en el presente estudio se han empleado un mayor número de tareas de TM, se espera encontrar resultados superiores en niñas que en niños en los distintos tipos sociométricos.

-Hipótesis 2. Según el estudio de Astington (1993) y Wellman (1990), leído en Slaughter y otros (2002), la TM va desarrollándose a medida que el proceso de sociabilización avanza. Por tanto, la edad implica una mejora en las habilidades de TM, y se espera encontrar mejores resultados en los niños/as de cursos superiores dentro de las distintas etapas de Primaria.

-Hipótesis 3. A partir de los estudios resumidos en la Tabla 1, es esperable que un niño rechazado demuestre un nivel de TM inferior que un niño Popular. Del mismo modo, se espera que cuanto menos oportunidades tenga el niño de aplicar y ejercitar su TM, menor sea esta capacidad, y se demuestre el siguiente patrón en función de la competencia observada: Ignorado $<$ Rechazado $<$ Medio $<$ Controvertido $<$ Popular. 
-Hipótesis 4. Por último, cuanto menos socializado esté el alumno (tipos ignorados y rechazados), se espera encontrar una percepción de dicha socialización menos ajustada a la realidad.

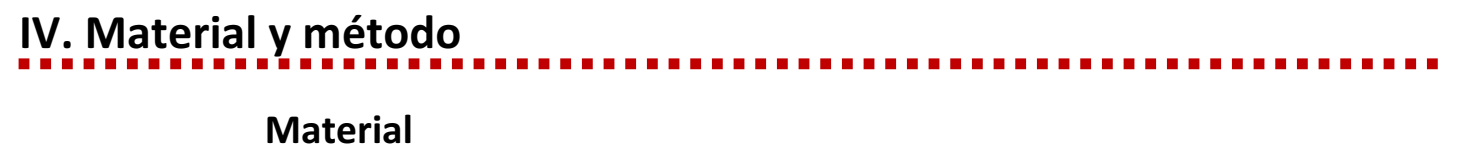

Medidas de relación entre iguales: valores sociométricos de los compañeros/as de aula. Para recoger medidas sociométricas de las dos aulas de Primaria se llevó a cabo el clásico método descrito por Coie y Dodge (1983), que consiste en preguntar a cada alumno/a de clase cuatro preguntas relacionadas con aspectos sociométricos: nominaciones (positivas y negativas) y percepciones (positivas y negativas). Para ello, el alumnado de cada aula cumplimentó un cuestionario respondiendo a las siguientes preguntas:

1. De todas las niñas y niños de tu clase, dime con quién te gusta estar mucho. ¿Por qué? ¿Con qué otros niños o niñas te gusta estar más? ¿Por qué? (Nominaciones Positivas: variable NP)

2. De todos los niños y niñas de tu clase dime con quién no te gusta estar. ¿Por qué? ¿Con qué otros niños o niñas no te gusta estar? ¿Por qué? (Nominaciones Negativas: variable NN)

3. De todas las niñas y niños de tu clase ¿A quién le gusta mucho estar contigo? ¿Por qué? ¿A qué otros niños o niñas les gusta mucho estar contigo? ¿Por qué? (Percepciones Positivas: variable PP)

4. De todas las niñas y niños de tu clase ¿A quién NO le gusta estar contigo? ¿Por qué? ¿A qué otros niños o niñas NO les gusta estar contigo? ¿Por qué? (Percepciones Negativas: variable PN)

Los datos del cuestionario sociométrico se analizaron con el software SOCIOMET (García-Bacete y González-Álvarez, 2010), con la ayuda del grupo Grupo de Investigación de Rechazo Entre Iguales en el Contexto Escolar (GREI) de la Universitat Jaume I. De éste análisis, se obtuvieron dos tipos de datos:

A. Valores sociométricos: NP, NN, PPA, PNA y FP. Sus definiciones pueden ser más ampliamente consultadas en el manual de uso SOCIOMET (García-Bacete y González-Álvarez, 2010): a) Nominaciones Positivas (NP): Es el número de veces que un alumno ha sido nominado positivamente por sus compañeros; b) Nominaciones Negativas (NN): Es el número de veces que un alumno ha sido nominado negativamente por sus compañeros; $c$ ) Percepciones positivas acertadas (PPA): Es un indicador del nivel de precisión del alumno al identificar o valorar los signos de aceptación o preferencia que emiten sus compañeros y del grado en que se ven cumplidas sus expectativas positivas; d) Percepciones negativas acertadas (PNA): Es un indicador, por una parte, del nivel de precisión del alumno en identificar o valorar los signos de rechazo o exclusión que emiten sus compañeros $y$, por otra parte, del grado en que se ven cumplidas sus expectativas negativas; e) Falsas percepciones (FP): Se trata de las percepciones emitidas, positivas y negativas, que no coinciden con las 
nominaciones recibidas. Es un indicador de expectativas sociales desajustadas o no cumplidas (FP = PP + PN - PPA - PNA).

$B$. Tipos sociométricos: cinco posiciones sociométricas dentro del aula (preferidos, rechazados, ignorados, controvertidos y medios).

Medidas de Teoría de la Mente (TM). Para realizar la evaluación de las habilidades mentalistas se han seleccionado aquellas acordes a primer y segundo ciclo de Primaria.

A. Falsa Creencia de $2^{\circ}$ orden (adaptación de Sullivan, Zaitchik y TagerFlusberg, 1994). Esta tarea pretende evaluar la habilidad de los niños y niñas para representarse creencias de segundo orden. Como en las tareas clásicas de primer orden, se narra una historia, pero en ésta dos personajes son independientemente informados de la nueva localización inesperada de un objeto (chocolate en la mochila). Se consideran de "2o orden" porque incluyen un segundo nivel de recursividad, es decir, una creencia de primer orden más una actitud proposicional de otra persona. Para la evaluación sólo se tendrán en cuenta dos de las preguntas de creencia de segundo orden: ¿Dónde piensa Judit que Sergio irá a buscar la tableta de chocolate? Realmente, ¿dónde está guardada la tableta de chocolate?. Para evaluar las respuestas, se puntuaron de la siguiente manera: 0 puntos: respuestas incorrectas (cuando la respuesta no es correcta); 1 punto: respuestas parciales (cuando la respuesta es correcta).

B. Historias Extrañas (Happé, 1994). La tarea de Historias Extrañas pretende evaluar la capacidad de comprender las intenciones comunicativas en diferentes situaciones cotidianas. De las 12 historias originales se eligieron dos, la mentira piadosa y la ironía, por estar relacionadas con la socialización entre iguales (Villanueva y otros, 2000). Para evaluar la comprensión de Historias Extrañas se se realizaron dos preguntas: a) Pregunta de comprensión: ¿Es cierto lo que dice...?. Con esta pregunta se intenta averiguar si comprenden que la frase no se puede interpretar en el sentido no literal; b) Pregunta mentalista: Entonces... ¿ Por qué... ha dicho eso? Con esta pregunta se pretende que los/las niños/as justifiquen su pregunta y que sean capaces de comprender el estado mental del según el contexto en el que sucede la historia. Las respuestas se puntuaron de la siguiente manera: 0 puntos: respuestas incorrectas (cuando no denota comprender la verdadera intención del protagonista y/o se queda con el sentido literal); 1 punto: respuestas parciales (cuando se intenta buscar una intencionalidad, pero no es la esperada); 2 puntos: respuestas correctas (cuando se reconocen y explican las intencionalidades correctas del hablante en el contexto)

C. Chistes gráficos. Esta prueba fue diseñada para evaluar el humor gráfico y la actividad representacional de los niños y niñas. Está basada en la adaptación de Andrés y Clemente (2010) sobre el trabajo de PucheNavarro (2004). En la tarea, hay un total de ocho chistes visuales, divididos en: 1) Chistes hiperbólicos: se caracterizan por el aumento desmesurado de algún elemento del dibujo; 2) Chistes mentalistas: se caracterizan porque la discrepancia "humorística" se encuentra en el pensamiento de los protagonistas; 3 ) Chistes de sustitución: algún elemento central (del 
objeto o protagonista) no está en concordancia con el resto (ej. Atribución de características humanas a animales); 4) Chistes complejos: cuentan dos o más transgresiones conjuntamente. Cada chiste visual estaba compuesto por tres viñetas: 1) Imagen lúdica; 2) Imagen sin atractivo lúdico; y 3) Imagen sin atractivo lúdico añadiendo un elemento distractor. Después de presentarse las tres opciones a la vez, el sujeto debe responder a la pregunta: ¿Cuál de las tres te parece más graciosa? (identificación).

D. Comprensión de emociones secundarias. Se administró una tarea de reconocimiento de emociones secundarias basada en estudios anteriores (Andrés y Clemente, 2010), en la que se valoró la comprensión de un total de siete emociones de acuerdo a la edad de la muestra: vergüenza, culpa, preocupación, orgullo, aburrimiento, celos/envidia y duda. Además, cada emoción se evaluó a través de dos modalidades: a) Expresión verbal (expresar cómo se siente el protagonista de la historia mediante palabras). Los participantes deben decidir cómo se siente el protagonista. Para ello, a continuación de la historia se pregunta: ¿Cómo se siente $X$ ? ; b) Identificación visual (señalar la expresión facial correspondiente). Los niños y niñas deben de identificar la cara más adecuada (entre 6 opciones) según la emoción que quiere transmitir con la ayuda de la narración y la ilustración. En este apartado también se les preguntó cómo se siente el niño: ¿Cómo se siente $X$ ?. La tarea se valoró de la siguiente manera: 0 puntos, si la emoción no era la correcta; 1 puntos si la emoción era la correcta.

E. Meteduras de pata. Esta tarea evalúa la capacidad de reconocer cuando un emisor dice algo sin mala intención, pero impacta negativamente en las emociones del interlocutor (Baron-Cohen y otros, 1999). Se emplearon dos situaciones en las que los participantes debían reconocer quién ha dicho algo que no debía, qué es lo que ha dicho que no debía haber dicho, y cómo ha hecho sentir a la otra persona. Estas iban combinadas con una ilustración en la que se mostraba lo que sucedía. A continuación, el sujeto debe identificar si se ha producido o no una metedura de pata y explicar en qué consiste contestando a siete preguntas ( 3 control de comprensión de la historia, y 4 sobre la metedura de pata). Para el estudio, sólo se tuvieron en cuenta cuatro implicadas en la metedura de pata: 1) ¿Quién ha dicho alguna cosa que no debería haber dicho?; 2) ¿Qué ha dicho que no debería haber dicho?; 3) ¿Quería _ hacer sentir mal a_? ; 4) ¿Cómo se sintió_ (la persona afectada)?.

En cuanto a la puntuación, las respuestas de los/las niños/as en la tarea se valoraron de la siguiente manera: 0 puntos: por cada respuesta no correcta; 1 punto: por cada respuesta correcta.

\section{Método}

Muestra. En este estudio participaron un total de 47 niño/as entre 7 y 10 años. Todos los/las niños/as pertenecían a un centro escolar público de un pueblo de la provincia de Castellón. El alumnado pertenecía a dos clases y etapas diferentes: 25 de ellos/ellas son de $2 \stackrel{0}{ }$ de primaria, Primera etapa 
(de 7 a 8 años, 14 chicos y 11 chicas), y 22 son de 4o de primaria, Segunda Etapa (9 a 10 años, 10 chicos y 12 chicas).

Procedimiento. Para realizar el presente trabajo de modo empírico, se solicitaron las autorizaciones necesarias en el centro. En cuanto a la administración de las pruebas, se realizaron en dos días. Primero se completó el cuestionario del estudio sociométrico. Se efectuó en cada aula (2 y 4 de Primaria) de manera colectiva en una sola sesión de 60 minutos. En las dos aulas se siguió el mismo procedimiento: se explicó la prueba de forma oral y, para asegurar la sinceridad de los participantes en sus respuestas. Una vez las dos clases habían realizado el cuestionario sociométrico, se procedió a evaluar la TM. Para garantizar que el alumnado entendía las instrucciones, cada clase se dividió en dos pequeños grupos (entre 11 y 13 personas). Se les proporcionó un dosier a cada uno/a con las pruebas mencionadas en el apartado anterior para evaluar sus habilidades de TM. Finalmente, se procedió a leer los enunciados y formular las preguntas oportunas para la realización de cada prueba. A estos enunciados se les acompañaba de unas ilustraciones proyectadas en una pizarra digital.

Tanto en el sociograma como en el test para evaluar la TM, se separaron los pupitres de manera que ninguno pudiera ver lo que contestaba el compañero/a para que fuese una actividad completamente individual y evitar lo máximo posible cualquier tipo de coacción entre ellos.

Tabla 2. Resumen de las tareas e instrumentos empleados en el estudio, y rangos de puntuación de cada una de ellas.

\begin{tabular}{|c|c|c|c|}
\hline Área & Medidas & Tareas/instrumentos & Variables y rango \\
\hline $\begin{array}{c}\text { Medidas } \\
\text { sociales }\end{array}$ & $\begin{array}{l}\text { Cuestionario } \\
\text { Sociométrico }\end{array}$ & Cuestionario y SOCIOMET & $\begin{array}{l}\text { VALORES: NP, NN, } \\
\text { PPA, PPN, FP. } \\
\text { TIPOS: Preferido, } \\
\text { ignorado, controvertido, } \\
\text { medio y rechazado. }\end{array}$ \\
\hline \multirow{5}{*}{$\begin{array}{l}\text { Medidas } \\
\text { de TM }\end{array}$} & $\begin{array}{l}\text { Creencia Falsa de } 2^{\circ} \\
\text { orden }\end{array}$ & Tarea de los hermanos & Rango: 0 -2 \\
\hline & $\begin{array}{l}\text { Intenciones } \\
\text { comunicativas }\end{array}$ & $\begin{array}{l}\text { Has Extrañas: ironía y mentira } \\
\text { piadosa }\end{array}$ & Rango: 0- 4 \\
\hline & Humor gráfico & $\begin{array}{l}\text { Tarea de chistes gráficos ( } 8 \\
\text { láminas) }\end{array}$ & Rango: 0 - 8 \\
\hline & Comprensión emocional & $\begin{array}{l}\text { Tarea de emociones secundarias } \\
\text { (verbal / visual) }\end{array}$ & $\begin{array}{l}\text { Rango: } \mathbf{0}-\mathbf{1 4} \\
-\quad \text { CE verbal: } 0-7 \\
-\quad \text { CE visual: } 0-7 \\
\end{array}$ \\
\hline & Meteduras de pata & $\begin{array}{l}\text { Tarea del pastel de manzana, y } \\
\text { Tarea del Avión (2) }\end{array}$ & Rango: 0 - 8 \\
\hline \multicolumn{3}{|c|}{ SUMA TOTAL TM } & Rango: 0 - 36 \\
\hline
\end{tabular}




\section{Resultados}

Los datos se analizaron Departamento de Psicología Evolutiva, Educativa, Social y Metodología de la UJI con ayuda del grupo GREI.

Estudio de la TM en función de la edad, sexo y tipo sociométrico.

Si se hace una comparación por sexo (Tabla 3), se observa en general que los chicos tienen una mejor TM tanto en el total como si se comparan las diferentes tareas de manera individualizada. Si se concreta a nivel de aula, se observa que las chicas tienen una mejor puntuación en la tarea de CF de segundo orden y en la tarea de emociones verbales y visuales, pero sólo en la clase de cuarto de primaria. En el aula de segundo, en la tarea de CF de segundo orden tienen la misma puntuación que los chicos. Y por lo que respecta al resto de habilidades de TM, en todas son superior los niños a las niñas. Es más, si se comparan directamente el total de las habilidades de la TM, tanto si los resultados se dividen por aulas o se comparan en conjunto, los niños siempre están por encima que las niñas.

Tabla 3. Comparación entre chicas y chicos según sexo y curso

\begin{tabular}{|c|c|c|c|c|c|c|}
\hline & $\begin{array}{l}\text { Emociones } \\
\text { Secundarias }\end{array}$ & $\begin{array}{l}\text { Historias } \\
\text { Extrañas }\end{array}$ & $\begin{array}{l}\text { Chistes } \\
\text { gráficos }\end{array}$ & $\begin{array}{l}\text { Meteduras de } \\
\text { pata }\end{array}$ & $\begin{array}{l}\text { CF segundo } \\
\text { orden }\end{array}$ & $\begin{array}{l}\text { Total } \\
\text { TM }\end{array}$ \\
\hline Chicos 2@P & 5,38 & 2,54 & 4,92 & 6,69 & 1,38 & 20,92 \\
\hline Chicas 2oP & 4,42 & 2,5 & 4,25 & 6,58 & 1,25 & 19,00 \\
\hline Chicos 4우 & 5,7 & 3,3 & 6,7 & 7,3 & 1,4 & 24,40 \\
\hline Chicas 4op & 5,92 & 3 & 6,25 & 6,92 & 1,25 & 23,33 \\
\hline Total Chicos & 11,08 & 5,84 & 11,62 & 13,99 & 2,78 & 45,32 \\
\hline Total Chicas & 10,34 & 5,5 & 10,5 & 13,5 & 2,5 & 42,33 \\
\hline
\end{tabular}

Si nos centramos en la edad los niños/as (Tabla 5), se observa que los niños de cuarto tienen mejor puntuación en todas las tareas excepto en la de CF de segundo orden, que tienen la misma.

En la Figura 1 se observa que los niños populares son los que han obtenido mejores resultados en las tareas de la TM seguidos de los medios y los que peor puntuación ha obtenido ha sido el ignorado seguido del rechazado. 

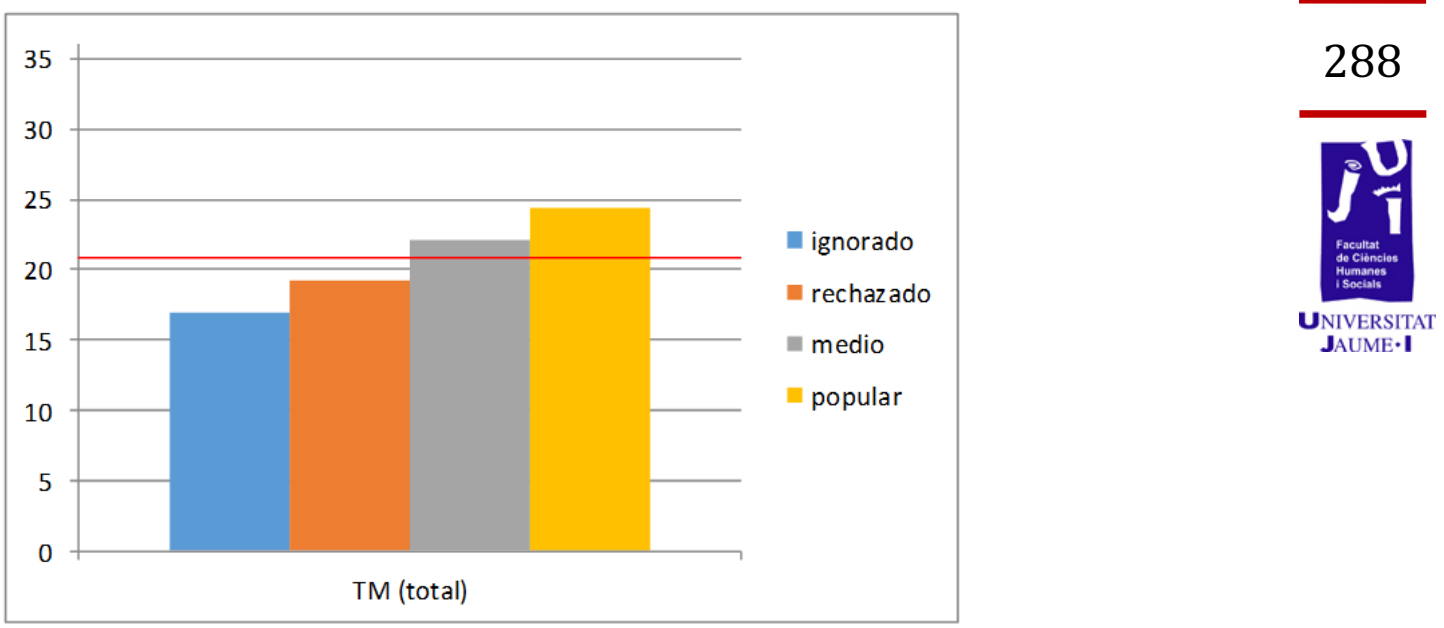

Figura 1. Relación entre Total de TM y los distintos Tipos Sociométricos

Al compararla la TM en función del tipo sociométrico (Tabla 4) de los alumnos los resultados son los siguientes: se observa una mayor competencia en todas las tareas de TM según el tipo sociométrico, siguiendo el patrón ignorado < rechazado < medio < popular. No se contempla el tipo "controvertido", puesto que en los datos del estudio no aparece ningún sujeto con esta condición.

Tabla 4. Relación entre chicos y chicas de los diferentes Tipos Sociométricos comparados con las medias de cada tarea.

\begin{tabular}{|c|c|c|c|c|c|c|c|c|c|c|c|c|c|c|c|c|c|c|}
\hline & $\begin{array}{l}\text { Em } \\
\text { Sec }\end{array}$ & $\begin{array}{l}\text { nocior } \\
\text { cunda }\end{array}$ & $\begin{array}{l}\text { nes } \\
\text { rias }\end{array}$ & & $\begin{array}{l}\text { stori } \\
\text { trañ }\end{array}$ & & & $\begin{array}{l}\text { hist } \\
\text { ráfic }\end{array}$ & & & $\begin{array}{l}\text { tedu } \\
\text { e pat }\end{array}$ & & CF & $\begin{array}{l}\text { segur } \\
\text { order }\end{array}$ & ado & & uma T & \\
\hline & $\mathrm{O}$ & A & $\mathbf{T}$ & 0 & $A$ & $T$ & 0 & A & $\mathbf{T}$ & 0 & A & $\mathbf{T}$ & 0 & A & $T$ & $\mathrm{O}$ & $A$ & $T$ \\
\hline Ignorado & 2 & 2 & 2 & 3 & 2 & 2,5 & 5 & 5 & 5 & 8 & 5 & 6,5 & 1 & 1 & 1 & 19 & 15 & 17 \\
\hline Rechazado & 5,5 & 4,3 & 4,8 & 1,3 & 1,5 & 1,4 & 6,3 & 5,5 & 6 & 5 & 7 & 5,8 & 1,3 & 1 & 1,2 & 18,3 & 20,5 & 19,2 \\
\hline Medio & 5,8 & 5,2 & 5,5 & 3,2 & 2,8 & 3 & 5,7 & 5,1 & 5,4 & 7,1 & 6,7 & 6,9 & 1,3 & 1,2 & 1,3 & 23,2 & 21,1 & 22,1 \\
\hline Popular & 6 & 6 & 6 & 2,7 & 3,5 & 3 & 5,3 & 6,5 & 5,8 & 7,7 & 7,5 & 7,6 & 2 & 2 & 2 & 23,7 & 25,5 & 24,4 \\
\hline $\begin{array}{c}\text { Promedio } \\
(d t)\end{array}$ & & $3(2$, & & & $8(1,2$ & & &, $5(1$, & & & $9(1,6$ & & & $3(0, i$ & & & $1,8(4$, & \\
\hline
\end{tabular}


A continuación, y dada la relevancia en las hipótesis del presente estudio, se procede a mostrar los datos de TM, comparando los tipos sociométricos en función del sexo. Tipo Ignorado/a: los niños ignorados tienen una mejor TM que las niñas ignoradas, sobre todo en las tareas de Metedura de pata e Historias Extrañas. En el resto, se ha obtenido el mismo valor. Y por lo que respecta al tipo Ignorado en conjunto entre chicas y chicos, la TM que muestran es inferior a la media respecto al resto de tipos. Tipo Rechazado/a: si se compara directamente las niñas con los niños de su mismo tipo, las niñas rechazadas tienen una mejor TM respecto a los niños rechazados, superándolos en todas las tareas menos en los chistes gráficos y la creencia de segundo orden. Los chicos tienen un nivel por debajo de la media en Historias Extrañas. Si se compara como un conjunto (chicas y chicos juntos). Tipo Medio/a: los chicos con el tipo sociométrico medios han obtenido mayor puntuación en todas las tareas, que las chicas con el tipo "medio". Tipo Popular: las chicas obtienen una mejor TM, superando a los chicos en las tareas de Historias Extrañas y los Chistes gráficos. Concretamente, han obtenido la misma puntuación en la tareas de emociones secundarias y de CF de segundo orden, mientras en el la tarea de Meteduras de pata han sido los chicos los que han sacado mejor puntuación.

\section{Percepciones positivas y negativas acertadas en función del tipo sociométrico.}

Como se observa en la Figura 2, los niños y niñas ignorados/as conforman el tipo sociométrico con menos percepciones positivas acertadas (PPA), seguidos de los rechazados y los medios. Por el contrario, los populares son más acertados en dicha percepción. Respecto a las percepciones negativas acertadas (PNA), se observa que en este caso, son los alumnos rechazados los que obtienen una puntuación más alta que el resto de tipos, siguiéndoles los medios, después los ignorados y por último los populares. Finalmente, en relación a las falsas percepciones (FP), los alumnos ignorados son con gran diferencia el grupo de niños que más falsas percepciones demuestran tener. El resto de tipos, manifiestan una cantidad de falsas percepciones más baja, y más o menos semejante. Por tanto, los sujetos ignorados son aquellos una mayor falsa expectativa de su socialización, puesto que son menos nominados de lo que esperan. 


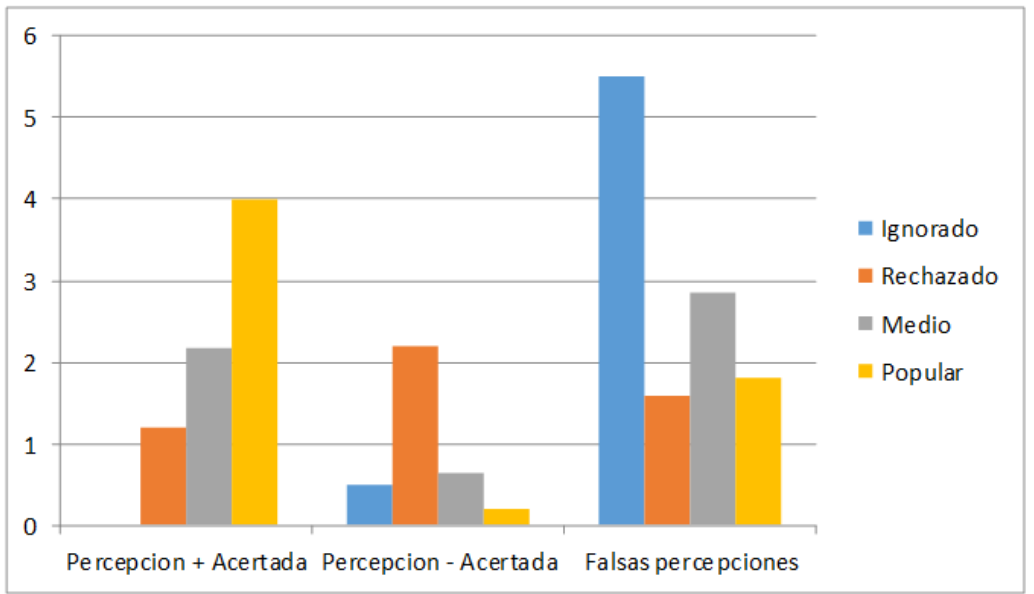

Figura 2. Relación entre Tipo Sociométrico y las diferentes percepciones.

\section{Conclusiones}

En el presente trabajo experimental se pretendía estudiar la TM de manera comprehensiva en distintas categorías de socialización entre iguales, teniendo en cuenta aspectos como el sexo y la edad de los alumnos, así como ampliar la información de estudios anteriores con medidas mentalistas como emociones secundarias o sentido del humor. Además, se pretendía analizar el ajuste a la realidad de su socialización. Para ello, se establecieron una serie de hipótesis, que los datos han permitido verificar.

En primer lugar, la primera hipótesis apostaba por encontrar un perfil superior en TM en las niñas respecto a los niños, independientemente de cuál fuera su tipo sociométrico. Los resultados del trabajo han corroborado parte de esta hipótesis. A nivel general, ambos grupos (chicas y chicos) han demostrado un nivel medio de TM semejante, ligeramente superior en el caso de los chicos. Si se observa esta diferencia en la ejecución para cada tipo sociométrico, solamente se encuentran diferencias para los tipos rechazado/a y popular, siendo las chicas rechazadas y populares, ligeramente superiores en su TM a los chicos rechazados y populares, respectivamente. Este dato confirma y está de acuerdo con en el estudio de Villanueva y otros (2000). Sin embargo, esta hipótesis no se cumplió para la categoría ignorado y medio, puesto que fueron aquí las niñas ignoradas y medias las que demostraron menor competencia en TM que los niños ignorados y medios, respectivamente.

En segundo lugar, atendiendo a las diferencias en la competencia mentalista esperable en función de la edad de los niños y niñas (primer ciclo vs segundo ciclo de primaria), se confirma que en efecto, la edad es un factor condicionante en la adquisición de la TM. Así, el alumnado de segundo ciclo de primaria demostró un nivel superior tanto en la variable de TM global, como en las distintas tareas por separado. Por tanto, se confirma que el desarrollo de la TM depende principalmente de un proceso 
de sociabilización rico entre iguales tal y como se demostraba en trabajos anteriores (Astington, 1993; Wellman, 1990, leído en Slaughter, V., Dennis, M. J., y Pritchard, 2002). Así, contra más edad, más oportunidades de entrenamiento social han tenido, $y$, los niños mayores son los que mejor nivel de TM tienen.

En tercer lugar, se esperaba encontrar diferencias entre las habilidades de TM entre los niños con distintos tipos sociométricos, constituyendo la hipótesis más relevante en este estudio. En la Tabla 5 se ofrece, a modo resumido e intentando completar la información de la Tabla 1 del apartado de la introducción, en qué tareas de TM ha obtenido niveles significativos (por encima o por debajo de la media \pm una desviación típica) cada tipo sociométrico. Cabe comentar que, posiblemente, con un aumento de la muestra, los resultados podrían haber aflorado más diferencias entre tipos en la mayoría de tareas.

Tabla 5. Resumen de los resultados del estudio sobre TM en distintos tipos sociométricos.

\begin{tabular}{|c|c|c|c|c|c|c|}
\hline & $\begin{array}{c}\text { Emociones } \\
\text { visual y verbal }\end{array}$ & $\begin{array}{c}\text { Historias } \\
\text { Extrañas }\end{array}$ & $\begin{array}{c}\text { Chistes } \\
\text { gráficos }\end{array}$ & $\begin{array}{c}\text { Meteduras de } \\
\text { pata }\end{array}$ & $\begin{array}{c}\text { CF segundo } \\
\text { orden }\end{array}$ & Suma TM \\
\hline Ignorado & $<$ media & $=$ media & $=$ media & $=$ media & $=$ media & $<$ media \\
\hline Rechazado & $=$ media & $=$ media & $=$ media & $=$ media & $=$ media & $=$ media \\
\hline Medio & $=$ media & $=$ media & $=$ media & $=$ media & $=$ media & $=$ media \\
\hline Popular & $=$ media & $=$ media & $=$ media & >media & $=$ media & $=$ media \\
\hline
\end{tabular}

En este sentido, en el presente estudio se demuestra que el alumnado "popular" tiene una mayor competencia en la globalidad de las tareas de TM administradas, de acuerdo con estudios los Slaughter y otros (2002), aunque su puntuación no superó una desviación típica por encima de la media. No obstante, sí muestran una habilidad significativa por encima de la media en la tarea de Meteduras de Pata. El control de esta habilidad, supone que tendrán mejor apreciación de lo que puede molestar o herir a sus compañeros, por tanto, es coherente que los populares reciban más nominaciones positivas puesto que saben cómo deben de tratar a otro igual. En el caso de los niños "ignorados", los datos del presente trabajo complementan a groso modo la visión de los estudios anteriores que habían prestado especial atención al tipo rechazado o popular. Concretamente, el dato más relevante del análisis llevado a cabo es que los alumnos ignorados presentaron una clara inferioridad al resto del alumnado en lo que a adquisición de TM se refiere. Es destacable que presentaron niveles muy por debajo de la media incluso en la tarea emocional. Así, con los resultados del presente estudio se amplían los estudios de Slaughter y otros (2002) o Peterson y Siegal (2002) poniendo 
en evidencia que son los sujetos Ignorados, y no especialmente los Rechazados, los que están por debajo de la media en la TM.

Una posible explicación a este hecho podría ser que el niño que es rechazado dispone de más oportunidades de sociabilizarse, ya sea positiva o negativamente, y de aprender de los errores de dichas interacciones a lo largo de su socialización. En este sentido, podrá pasar tiempo pensando en las consecuencias de sus actos, cómo se sienten sus compañeros o qué piensan de él. Sin embargo, el niño que es ignorado u olvidado, no interacciona (para bien ni para mal) con el resto del grupo, simplemente no se acuerdan del él/ella. Probablemente juegue solo, no interactúe con sus iguales en clase, en el patio ni en actividades de grupo, y en consecuencia, pierde oportunidades para practicar sus habilidades mentalistas, les cueste predecir por qué hacen/dicen unas cosas u otras; etc.

Finalmente, se pretendía averiguar si las percepciones que el alumnado tiene de su estatus en el aula son más o menos acertadas y ajustadas a la realidad en función del tipo sociométrico al que pertenecen. Se han sacado conclusiones muy interesantes y novedosas respecto a los estudios vistos anteriormente sobre todo en relación a las falsas percepciones y las nominaciones inesperadas tanto positivas como negativas. Por un lado, respecto las falsas percepciones, se ha observado que son los niños ignorados los que más falsas expectativas sobre su propia socialización tienen en comparación al resto de grupos. Parece que estos niños creen que sus compañeros les eligen (bien de forma positiva o bien de forma negativa), pero en realidad no es así, por tanto todas las expectativas que tiene son erróneas o falsas. Por otro lado, otro dato a destacar es el patrón observado en las nominaciones inesperadas: los niños rechazados esperan obtener menos nominaciones negativas de las que reciben (coherente con su estatus de rechazo dentro de su aula) y los populares menos positivas de las que reciben (coherente con su estatus de popularidad en el aula). Parece que el sujeto que es rechazado tiene una expectativa de la realidad más distorsionada, puesto que parece no ser consciente de que los demás no quieren estar con él. $Y$, espera que haya algunos compañeros de aula quiera estar con él/ella, no espera que sean tantos. Contrariamente, el alumnado popular, como es esperable, recibe una cantidad de nominaciones inesperadas positivas mucho más elevada de la esperada, puesto que seguramente sea consciente de que es querido por unos cuantos alumnos del aula, lo más probable es que no perciba que es la mayoría quiere estar con él.

Llegado a este punto, se puede afirmar que los objetivos de esta investigación se han cumplido. Por una parte se ha investigado sobre la TM de manera comprehensiva teniendo en cuenta factores como la edad, el sexo y los tipos sociométricos de los alumnos. Todas estas variables han sido de gran utilidad para entender mejor las habilidades mentalistas del alumnado de Primaria y, por otra parte, para poder ampliar parte de la información de estudios previos. El uso de las variables sociométricas (nominaciones y percepciones) ha sido muy importante para poder 
conocer la visión de la realidad que tienen los distintos alumnos de ellos mismos respecto a sus compañeros. Sin embargo, en esta investigación no se ha trabajado de manera individual, sino que los niños han sido agrupados por tipo sociométrico para estudiar si se cumplía el patrón ignorado $<$ rechazado $<$ medio $<$ popular.

En estudios anteriores ya se habían evidenciado ciertos sesgos y dificultades para los niños rechazados en el desarrollo de su TM y, en este sentido, el presente estudio aporta evidencias sobre la TM de los niños ignorados. Así, se demuestra que el niño ignorado, que carece del proceso de sociabilización tanto negativo como positivo, está en riesgo de no desarrollar tan rápidamente como sus iguales sus habilidades mentalistas. Con este dato, se barajan posibles puntos de vista acerca de la construcción social de la TM: ¿realmente es la TM la que conlleva que un niño/a tenga un tipo sociométrico determinado o, por el contrario, es el impacto social del niño en el grupo el que conlleva un mejor o peor desarrollo de las habilidades mentalistas? Futuras investigaciones podrían intentar seguir desvelando dicha incógnita.

El rechazo escolar, como ya se ha comentado a lo largo del artículo, es un gran problema al que cada vez se le está haciendo más hincapié. En el aula, el/la niño/a puede verse excluido/a entre sus iguales o compañeros, e incluso puede llegar a influir en la actitud del profesorado (García-Bacete, Sureda y Monjas (2010). Estos/as niños/as representan rasgos generales como un escaso nivel de actividad social, una menor autoestima, disfrutan menos de las actividades de clase, se muestran insatisfechos con las relaciones de profesores y compañeros y con las normas de clase, etc. (ver revisión en García-Bacete y otros, 2010). Este problema puede tener graves consecuencias en el futuro tanto académico como social en el niño/a, y por ello resulta esencial su detección temprana para dar una respuesta de intervención que intente paliar este fenómeno lo antes posible.

En este sentido, una posible intervención temprana podría consistir en prevenir o disminuir el porcentaje de rechazo escolar entre iguales a partir de la mejora sus habilidades mentalistas. Concretamente, y en relación al diseño de dicha intervención, se podría intentar mejorar las habilidades mentalistas en los alumnos/as mediante actividades en las que intervengan ellos y sean los propios protagonistas. Unas pequeñas representaciones teatrales, por ejemplo, en el que tengan que trabajar distintos personajes, representar distintas emociones y ponerse en el papel tanto de su propio personaje como del resto que intervienen en la historia. Además, para mejorar la preferencia e impacto social en el aula, se podría hacer intervenciones enfatizando la cooperación entre iguales, ya sea mediante trabajos, actividades o tutorías.

Últimamente, cabe mencionar que sería idóneo como líneas de futuro continuar esta investigación haciendo un seguimiento de los mismos niños a través de la etapa de Primaria, es decir, hacer un diseño longitudinal. Podría mantenerse el mismo método y así determinar si los tipos sociométricos que se han dado en las dos aulas se mantienen, se acentúan o han cambiado; así como si el desarrollo de la TM sigue evolucionando 
acorde con los resultados obtenidos, o, transcurridos los años, los niveles de los niños ignorados, por ejemplo, mejoran o disminuyen.

\section{Bibliografia}

Andrés-Roqueta, C., Clemente-Estevan, R.A., Cuervo-Gómez, K., y GórrizPlumed, A. (2009). << ¿Necesita Superman alas para volar? Comprensión del humor gráfico en niños y niñas con Trastorno Específico del Lenguaje. > International Journal of Developmental and Educational Psychology, 1(3), 257-266.

ASHER, S. R., Y WHEELER, V. A. (1985). «< Children's loneliness: a comparison of rejected and neglected peer status. $>>$ Journal of consulting and clinical psychology, 53(4), 500.

Baron-Cohen S, O'Riordan M, Stone V, Jones R, Plaisted K. <<Recognition of faux pas by normally developing children and children with Asperger syndrome or high-functioning autism $\gg$, J Autism Dev Disord 1999a; 29: 407-18.

COIE, J. D., Y DODGE, K. A. (1983). « Continuities and changes in children's social status: A five year longitudinal study >>, Merrill-Palmer Quarterly, 29, 261-282.

García Bacete, F. J., Sureda García, I., y Monjas Casares, M. I. (2010). « El rechazo entre iguales en la educación primaria: Una panorámica general $\gg$, Anales de psicología, 26(1), 123-136.

García-Bacete, F. J., y González-ÁlvareZ, J. (2010). Evaluación de la competencia social entre iguales. TEA Ediciones, Madrid.

HAY, D. F., PAYNE, A., Y CHADWICK, A. (2004). <<Peer relations in childhood >>, Journal of Child Psychology and Psychiatry, 45(1), 84-108.

HugheS, C., y LEEKAM, S. (2004). <<What are the links between theory of mind and social relations? Review, reflections and new directions for studies of typical and atypical development $\gg$, Social Development, 13(4), 590-619.

Perner, J., Y Wimmer, H. (1985). <<John thinks that Mary thinks that: attribution of second order beliefs by 5 -year-old to 10 -year-old children $\gg>$, J Exp Child Psychol, 39, 437-71.

Puche Navarro, R. y Lozano, H. (2002). El Sentido del humor en el niño. Bogotá. Siglo del Hombre Editores.

PuChe-NAVARRO, R. (2004). <<Graphic jokes and children's mind: An unusual way to approach children's representational activity $>>$, Scandinavian Journal of Psychology 45 (4), 343-355. 
RoquetA, C. A., y Estevan, R. A. C. (2010). <<Dificultades pragmáticas en el trastorno específico del lenguaje. El papel de las tareas mentalistas $\gg$, Psicothema, 22(4).

Slaughter, V., DenNis, M. J., y PRITChARd, M. (2002). <<Theory of mind and peer acceptance in preschool children $>>$, British Journal of Developmental Psychology, 20(4), 545-564.

Sullivan, K., ZAITCHIK, D., y TAGeR-FlusberG, H. (1994). «<Preschoolers can attribute second-order beliefs >>, Developmental Psychology, 30(3), 395.

Villanueva, L. V., Clemente, R. A., y García-BAcete, F. J. (2000). <<Theory of mind and peer rejection at school >, Social Development, 9(3), 271-283. 